\title{
Evaluation of Antioxidant Activity of Tea Polyphenols by a Quantum Chemistry Calculation Method - PM6
}

\author{
Qing Meng ${ }^{1}$, Ziyin Yang ${ }^{2}$, Guoliang $\mathrm{Jie}^{1}$, Ying $\mathrm{Gao}^{1}$, Xinghai Zhang ${ }^{3}$, Wei $\mathrm{Li}^{4}$, Bo $\mathrm{Li}^{1{ }^{1, *}}$, Youying $\mathrm{Tu}^{1{ }^{1, *}}$ \\ ${ }^{1}$ Department of Tea Science, Zhejiang University, Hangzhou, China \\ ${ }^{2}$ Key Laboratory of Plant Resource Conservation and Sustainable Utilization, South China Botanical Garden, Chinese Academy of \\ Sciences, Guangzhou, China \\ ${ }^{3}$ Department of Applied Engineering, Zhejiang Economic and Trade Polytechnic, Hangzhou, China \\ ${ }^{4}$ Department of Tea Culture, Zhejiang Shuren University, Hangzhou, China \\ *Corresponding author: youytu@zju.edu.cn (Y. Tu), drlib@zju.edu.cn (B. Li)
}

Received November 10, 2014; Revised November 17, 2014; Accepted November 21, 2014

\begin{abstract}
Tea polyphenols arepowerful natural antioxidants. To quickly evaluate theantioxidantactivity of these compounds, a quantum chemistry calculation method - PM6 was employed to calculate the heat of formation (HOF). The HOF valuewas negatively correlated well $(r=-0.910$, RMS $=0.12)$ with the antioxidant activity of flavanols (catechins), flavonols (kaempferol, quercetin, and myricetin), flavanones (dihydromyricetin), anthocyanins (delphinidin, cyanidin, pelargonidin), proanthocyanidins and theaflavins. The number and position of hydroxyl, and the structural groups influencing the conjugation between the B- and C-rings could affect the HOF value and radical scavenging activity of flavonoids. Therefore, the HOF value could be used as a descriptor for modeling antioxidant activity of polyphenols.
\end{abstract}

Keywords: antioxidantactivity, tea polyphenols, HOF, PM6

Cite This Article: Qing Meng, Ziyin Yang, Guoliang Jie, Ying Gao, Xinghai Zhang, Wei Li, Bo Li, and Youying Tu, "Evaluation of Antioxidant Activity of Tea Polyphenols by a Quantum Chemistry Calculation Method - PM6." Journal of Food and Nutrition Research, vol. 2, no. 12 (2014): 965-972. doi: 10.12691/jfnr-2-12-17.

\section{Introduction}

Free radicals have very close relationship with diseases, and play an essential role in the oxidative deterioration of food $[1,2]$. It is of great interest to scavenging redundant free radicals for human health and food storage. At present, the resources of antioxidant are mainly divided into natural products and the synthetic materials. The natural antioxidants with high efficiency and low toxicity have become more and more popular [3,4]. Numerous studies have reported that tea polyphenols are powerful natural antioxidants [5-10]. It's known that phenol hydroxyl $(\mathrm{PhOH})$ contributes a lot to theantioxidant activity of polyphenols $[11,12]$. The essential mechanism is that phenol hydroxyl reacts to ROS (Reactive Oxygen Species) such as the superoxide anion radical $\mathrm{O}^{-2}$, hydroxyl radical $\bullet \mathrm{OH}$, and lipid peroxyl radical LOO•, and form a stable semiquinone-type radicals to terminate the chain action: $\mathrm{PhOH}+\mathrm{R}^{\cdot} \rightarrow \mathrm{PhO}^{\circ}+\mathrm{RH}[13]$.

Numerous studies have been conducted to establish the relationship between flavonoid structure and their radical scavenging activity with quantum chemical calculations [14]. However, many molecules of similar structure don't obey these models [15]. PM6 (parameterized model 6) is a significant improvement and is much more precise than the previously used AM1 (Austin Model 1) and PM3 methods $[16,17,18]$. PM6 method in the MOPAC $2009^{\mathrm{TM}}$ software package is implemented in Semi- empirical Quantum Chemical Calculations (SQCC). Compared with DFT (Density Functional Theory) method, SQCC can save computer-time, especially in the case of larger molecules such as theaflavins. Many parameters calculated by PM6 method, such as molecular geometry, heat of formation (HOF), dipole moments, and dissociation energies, coincide well with the experimental results. Previous studies indicated PM6 was an efficient method to evaluate the antioxidant activity of flavonoids based on bond dissociation enthalpy (BDE) of $\mathrm{OH}$ groups [19]. The HOF value is associated with the ability of polyphenols to donate hydrogen atom, and was found to correlate well with the O-H BDE [20,21]. In this study, $\mathrm{HOF}$ values ofthe major series of polyphenols in tea were calculated by the PM6 method, and were used to predict the antioxidant activity of these compounds.

\section{Computational Details}

All calculations were performed using the MOPAC $2009^{\mathrm{TM}}$ program package with the method PM6. The HOF value was calculated by the following equation:

$$
\mathrm{HOF}=\mathrm{E}_{\text {elect }}+\mathrm{E}_{\text {nuc }}+\Sigma \mathrm{E}_{\text {isol }}(\mathrm{A})+\Sigma \mathrm{E}_{\text {atom }}(\mathrm{A})
$$

Where $E_{\text {elect }}$ is the electronic energy of the final materials, $E_{\text {nuc }}$ is the energy of internuclear repulsion in the final materials, $\Sigma \mathrm{E}_{\text {isol }}(\mathrm{A})$ is the energy required to 
remove all the valence electrons from the atoms in the system to an isolated state, and $\Sigma E_{\text {atom }}(\mathrm{A})$ is the energy required to isolate the atoms from their standard state to free state [22,23].

\section{Results and Discussion}

Flavanols, flavonols, flavanones, anthocyanins, proanthocyanidins and theaflavins are major polyphenols intea.In the present work, The HOF values of these compounds were calculated by the PM6 method, and the relevance between the HOF value and antioxidant activity of compounds was studied for the first time.

Two structural features of flavonoids can influence their antioxidant potential. The first is the number, position and pattern of hydroxyl substitution in the molecular, and the second is the presence of structural groups required for extended conjugation between the Band C-rings [11]. It is known that the $\mathrm{PhOH}$ on the $\mathrm{B}$ ring of flavonoids has strongest radical scavenging ability, and that on the A ring is the weakest. The antioxidant activity of pyrogallol is stronger than that of catechol [24].

Catechins, the primaryflavanols in tea, account for 70 $80 \%$ of tea polyphenols. They mainly consist of epicatechin (EC), epigallocatechin (EGC), epicatechingallate (ECG) and epigallocatechingallate (EGCG). The HOF values and antioxidant activityof the four catechins were presented in
Table 1. These antioxidant data were from previous literatures [11,25,26], and were determined bythe1,1diphenyl-2-picrylhydrazyl(DPPH), superoxide radical scavenging activity (SRSA) and troloxequivalent antioxidant capacity(TEAC)-ABTS assays.The order of HOF value was EGCG $<$ ECG $<$ EGC $<$ EC, which was opposed to their antioxidant activity sequence. These results showed that the lower HOF value of catechins, the stronger antioxidant activity. As shown in Figure 1, EGC has one more hydroxyl substituent than EC, and EGCG possesses one more C5'-OH than ECG in the B-ring. ECG and EGCG have a pyrogallol moiety in the C3 position. An increased number of $\mathrm{OH}$ groups could be related to the increased ability of $\mathrm{H}$ atom abstraction and increased scavenging of free radicals.

Table 1. Antioxidant activities of various catechins in different assays and their HOF values

\begin{tabular}{ccccc}
\hline $\begin{array}{c}\text { Tea } \\
\text { catechins }\end{array}$ & DPPH $[\mu \mathrm{M}]^{\mathrm{a}}$ & ${\text { SRSA }[\mu \mathrm{M}]^{\mathrm{b}}}^{\mathrm{T}}$ & $\left.\mathrm{TEAC}_{\mathrm{mmM}}\right]^{\mathrm{c}}$ & $\begin{array}{c}\mathrm{HOF} \\
{[\mathrm{kcal} / \mathrm{mol}]}\end{array}$ \\
\hline EC & 2.2 & $203 \pm 14$ & 3.16 & -221.56 \\
EGC & 1.1 & $32.3 \pm 1.4$ & 3.86 & -262.07 \\
ECG & 0.7 & $7.2 \pm 0.9$ & 4.23 & -356.80 \\
EGCG & 0.6 & $4.60 \pm 0.43$ & 4.39 & -401.82 \\
\hline
\end{tabular}

${ }^{a} \mathrm{DPPH}$ radical $50 \%$ Scavenging Concentration( $\left.\mathrm{IC}_{50}\right)$ (Nanjo et al., 1996).

${ }^{\mathrm{b}}$ Concentration of catechins for $25 \%$ inhibition of superoxide radical $\mathrm{O}_{2}$. in non-enzymatic system ( $\mathrm{IC}_{25}$ ) (Furuno et al., 2002). 'TEAC Value (Cai et al., 2006)

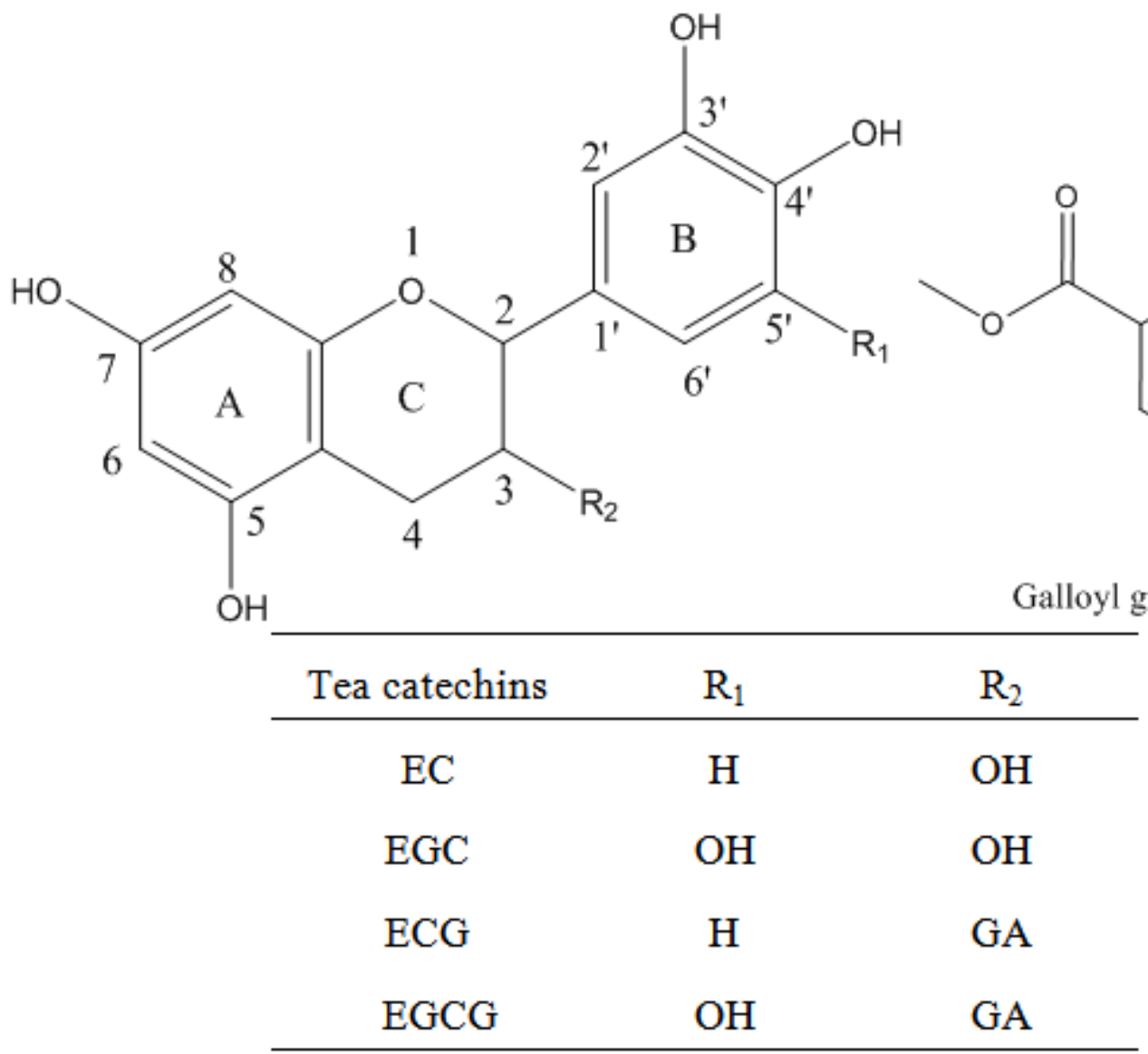

Figure 1. Structures of the tea catechins

Anthocyaninsmainly distributed in shoots of tea plant and account for the bitter taste of tea. Previous studies showed that pelargonidin, cyanidin and delphinidinwere the major anthocyanins in tea [27]. As shown in Table 2, the HOF value order was delphinidin < cyanidin < pelargonidin, and the antioxidant potential sequence of anthocyanins determined by SRSA and TEAC assays was delphinidin $>$ cyanidin $>$ pelargonidin $[28,29]$. These results 
suggested that higher antioxidant ability of anthocyanins is associated with lower HOF value. The structure difference of the three anthocyanins is the number and position of hydroxyl at B-ring. Delphinidin possesses three hydroxyls at $\mathrm{C}^{\prime}, 4^{\prime}$ and $5^{\prime}$ positions, cyanidinhas two hydroxylsat $\mathrm{C}^{\prime}$ and $4^{\prime}$ positions, and pelargonidin possesses onehydroxyl at $\mathrm{C}^{\prime}$ ' position in the B-ring (Figure 2). These result indicated HOF could serve as a theoretical measure for ranking anthocyanins as antioxidants.
Table 2. Antioxidant activities of various anthocyanins in different assays and their HOF values

\begin{tabular}{cccc}
\hline Anthocyanins & ${\text { SRSA }[\mu \mathrm{M}]^{\mathrm{a}}}$ & $\left.\mathrm{TEAC}^{\mathrm{m} M}\right]^{\mathrm{b}}$ & $\mathrm{HOF}[\mathrm{kcal} / \mathrm{mol}]$ \\
\hline Pelargonidin & 420 & $1.30 \pm 0.1$ & -155.67 \\
Cyanidin & 22 & $4.4 \pm 0.12$ & -196.63 \\
Delphinidin & 2.4 & $4.44 \pm 0.11$ & -239.47 \\
\hline
\end{tabular}

${ }^{\mathrm{a} C}$ Concentration of anthocyanins for $50 \%$ inhibition of superoxide radical O2.' (IC ${ }_{50}$ ) (Noda et al., 2002). ' ${ }^{\mathrm{b}} \mathrm{TEAC}$ value(RiceEvanset al., 1996).

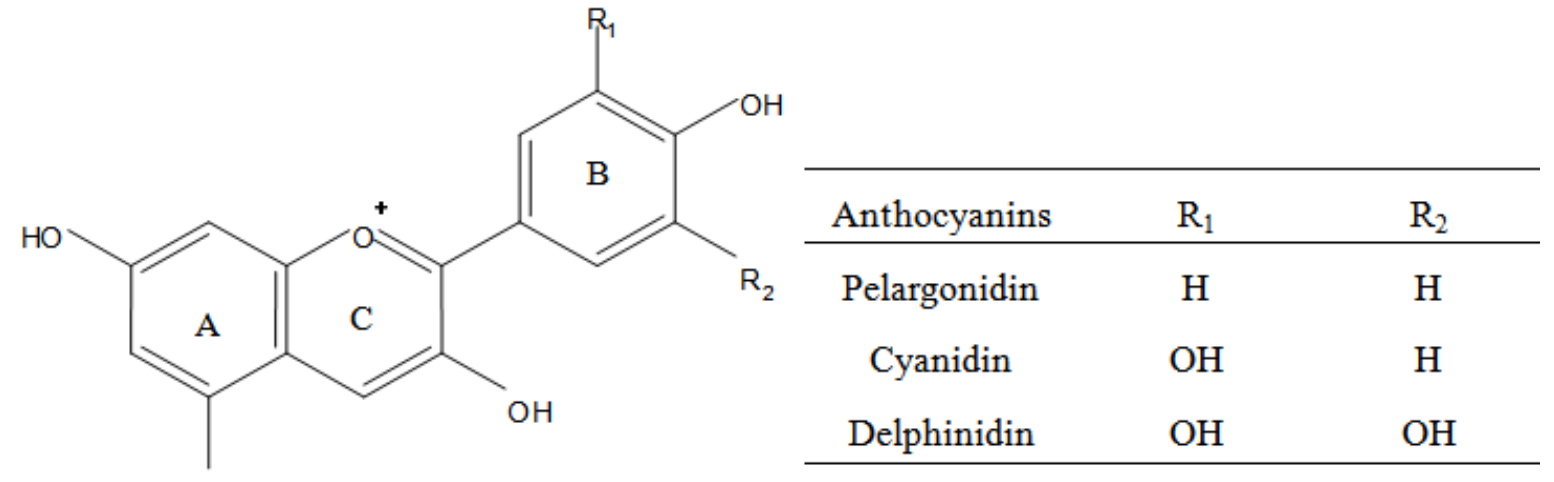

Anthocyanin

Figure 2. Structures of the anthocyanins

Tea contains more than 20 kinds of flavonols, which are considered to account for tea infusion color [30]. Our data showed the HOF values of kaempferol, quercetin and myricetin were $-179.36,-220.23$ and $-262.53 \mathrm{kcal} / \mathrm{mol}$, respectively. Figure 3 showed that the three flavonols have different amount of hydroxylin the B-ring, indicating that more hydroxylin the B-ring, lower HOF value. It was reported the activity of scavenging hydroxyl ( $\mathrm{OH})$ radicals decreases in the order: myricetin $>$ quercetin $>$ kaempferol [31], which meant the negative correlation existed between HOF and antioxidant activity of flavonol.<smiles>[R]c1cc(-c2oc3cc(O)cc(O)c3c(=O)c2O)cc([R])c1O</smiles>

Flavonol

\begin{tabular}{ccc}
\hline Flavonols & $\mathrm{R}_{1}$ & $\mathrm{R}_{2}$ \\
\hline kaempferol & $\mathrm{H}$ & $\mathrm{H}$ \\
quercetin & $\mathrm{H}$ & $\mathrm{OH}$ \\
myricetin & $\mathrm{OH}$ & $\mathrm{OH}$ \\
\hline
\end{tabular}

Figure 3. Structures of oligomericproanthocyanidins in tea and their HOF values

Flavanones are another type of flavonoids which contribute to the antioxidant activity of tea [32]. In order to explore the relationship between antioxidant ability and structure except for hydroxyl, EGC and dihydromyricetin were selected because they possess the same number and location of hydroxyl substitute. The HOF values of EGC and dihydromyricetin calculated in this study are -262.07 and $-286.22 \mathrm{kcal} / \mathrm{mol}$, respectively (Figure 4). Furthermore, we used frontier molecular orbital theory to analyze the energy difference $\triangle E\left(\triangle E=E_{\mathrm{LUMO}}-\mathrm{E}_{\mathrm{HOMO}}\right)$ of the two compounds. The $\triangle \mathrm{E}$ of EGC and dihydromyricetin is 12.99 and $7.16 \mathrm{eV}$, respectively, indicating dihydromyricetin is less stable and easier deprotonated than EGC [33]. Active electron orbital of EGC mainly focuses on B-ring, whereas that of dihydromyricetin distributes in the C4- carbonyl moiety, C2-3 bond, C3-OH group and adjacent hydroxyl substitute on the A and B-rings (Figure 5). Previous study showed that dihydromyricetin had stronger antioxidant potential than EGC [34]. Compared with EGC, dihydromyricetin contains a carbonyl moiety at $\mathrm{C} 4$, which is considered to be an essential structural requirement for flavonoid aroxyl radical stabilization [35,36]. The chromone structure in C-ring can enhancethe dissociation of hydroxyl of C-ring, and promote hydrogen generation of the C3-alcohol hydroxide [12]. In addition, the altered bonding in $\mathrm{C}$ ring allows delocalization between the $\mathrm{A}$ and $\mathrm{B}$ rings, thus stabilize the aryloxyl radical after hydrogen donation [37,38,39]. All these results may explain for the lower HOF value and higher antioxidant potential of dihydromyricetin compared with EGC. 
<smiles>Oc1cc(O)c2c(c1)OC(c1cc(O)c(O)c(O)c1)C(O)C2</smiles><smiles>O=C1c2c(O)cc(O)cc2OC(c2cc(O)c(O)c(O)c2)C1O</smiles>

Figure 4. Structures of the major flavonols in tea
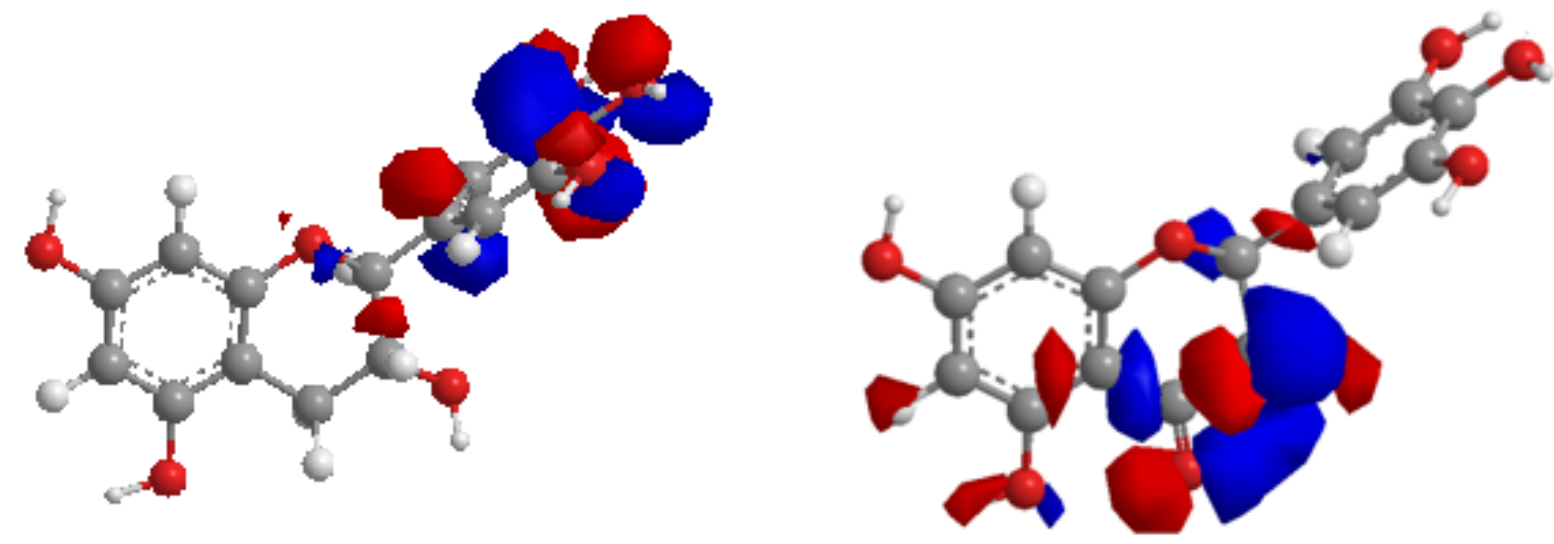

\section{$\mathrm{EGC} \mathrm{HOMO}=-10.71$ eVDihydromyricetin $\mathrm{HOMO}=-10.31 \mathrm{eV}$}
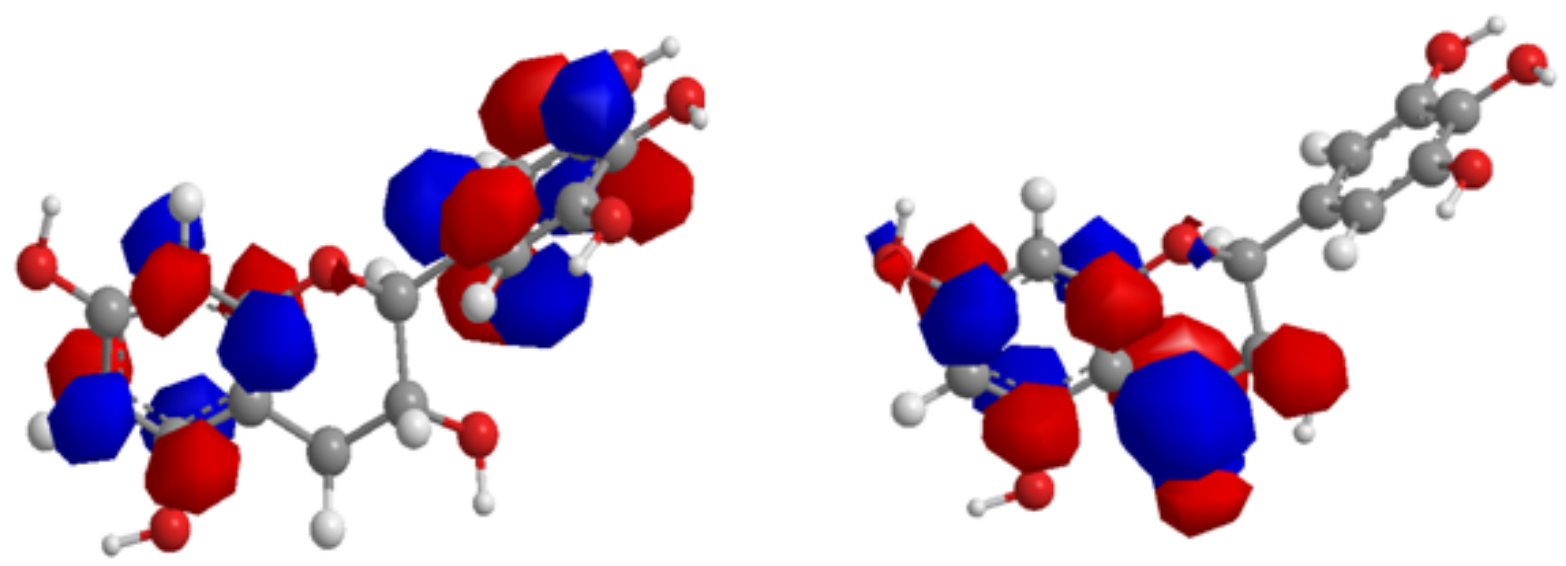

\section{EGC LUMO $=2.28$ eVDihydromyricetin $L U M O=-3.15 \mathrm{eV}$}

Figure 5. Structures of EGC and dihydromyricetin and their HOF values

In addition to the polyphenol monomers, the relevance between HOF value and antioxidant activity of polymers was studied in this work.Our data showed the HOF value sequence was quercetin-Fe complex (3:1)<quercetin-Zn complex $\quad(2: 1)<$ quercetin, indicating the order of antioxidant activity was quercetintrimer $>$ dimer $>$ monomer. This prediction was consistent with the previous antioxidant data determined by SRSA assay in Table 3 [40].
Table 3. The inhibition rates of quercetin and quercetin-metal complexes on $\mathrm{O}_{2}$-radical (Liet al., 2006)

\begin{tabular}{ccccccc}
\hline \multirow{2}{*}{ Material } & \multicolumn{5}{c}{ Concentration (units: $\times 10^{-5} \mathrm{~mol} / \mathrm{L}$ ) } \\
\cline { 2 - 7 } & 2.5 & 5.0 & 6.2 & 7.5 & 10 & 12.5 \\
\hline Quercetin & 25.4 & 37.3 & 33.3 & 40.5 & 52.4 & 54.8 \\
Quercetin-Zn(2:1) & 43.6 & 55.6 & 56.0 & 56.9 & 57.4 & 69.8 \\
Quercetin-Fe (3:1) & 57.1 & 69.8 & 72.5 & 80.2 & 88.9 & 94.4 \\
\hline
\end{tabular}


Proanthocyanidins (PAs) are a class of colorless phenolics characterized by an oligomeric or polymeric structure based on flavan-3-ol units. Flavan-3-ol units are connected via $\mathrm{C} 4 \rightarrow \mathrm{C} 8, \mathrm{C} 4 \rightarrow \mathrm{C} 6, \mathrm{C} 2 \rightarrow \mathrm{O} 7$ or $\mathrm{C} 2 \rightarrow \mathrm{O} 5$ linkages [41]. EC is considered to be one of most common constitutive units of Pas [27]. Our results showed the HOF value of PAs decreased with the rise ofdegree of polymerization (Figure 7). It was reported that the antioxidant activity of PAs largely depends on the degree of polymerization. Although the antioxidant activity of monomers was inferior to that of oligomers, the antioxidant ability of polymers reduced with the increased degree of polymerization. High polymerization degree may attenuate the dissociation of hydroxyl due to the existence of steric hindrance [42,43]. These results indicated the HOF value may only applies to oligomeric proanthocyanidins.

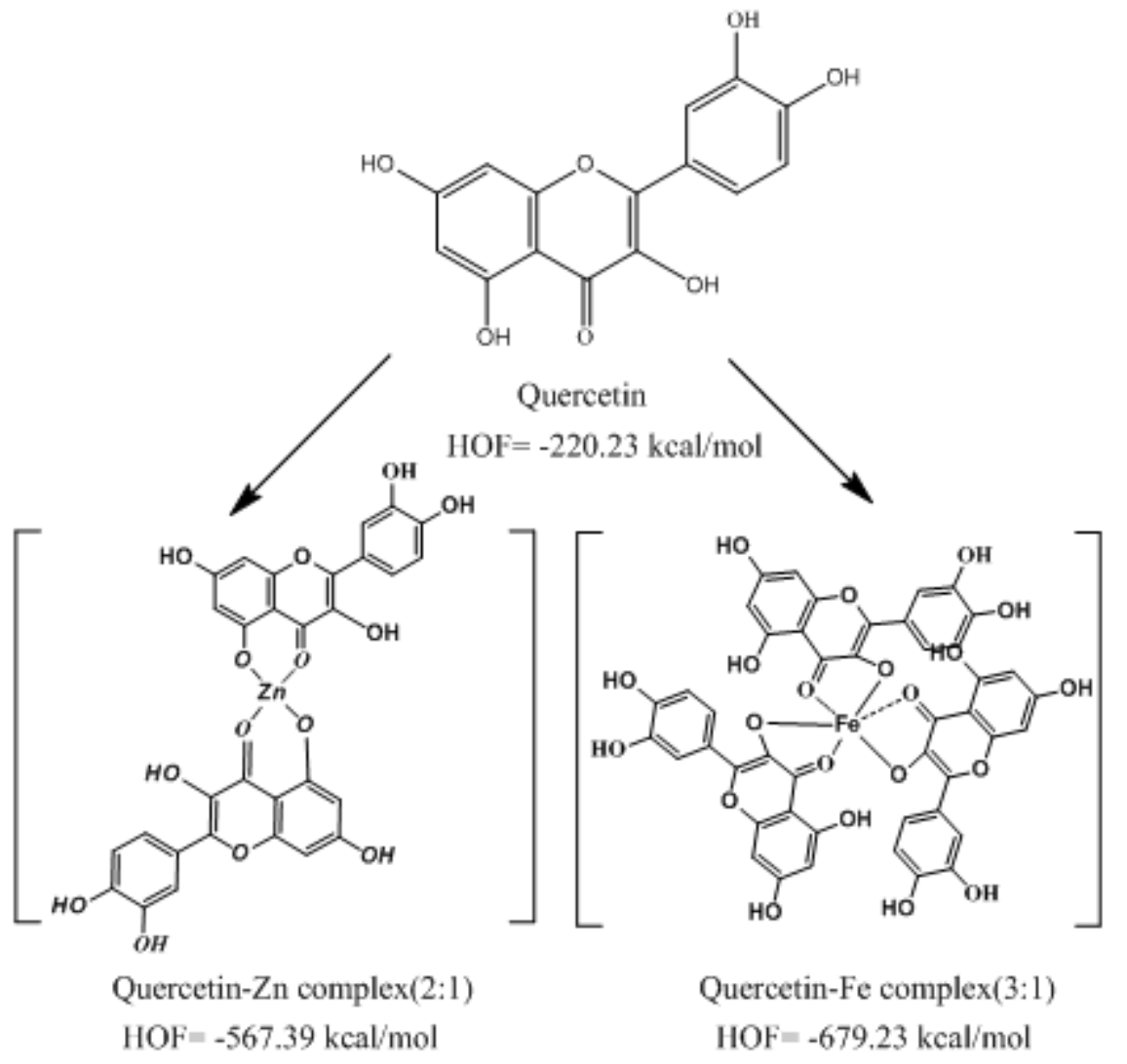

Figure 6. Highest Occupied Molecular Orbital (HOMO) and Lower Unoccupied Molecular Orbital (LUMO) of EGC and dihydromyricetin

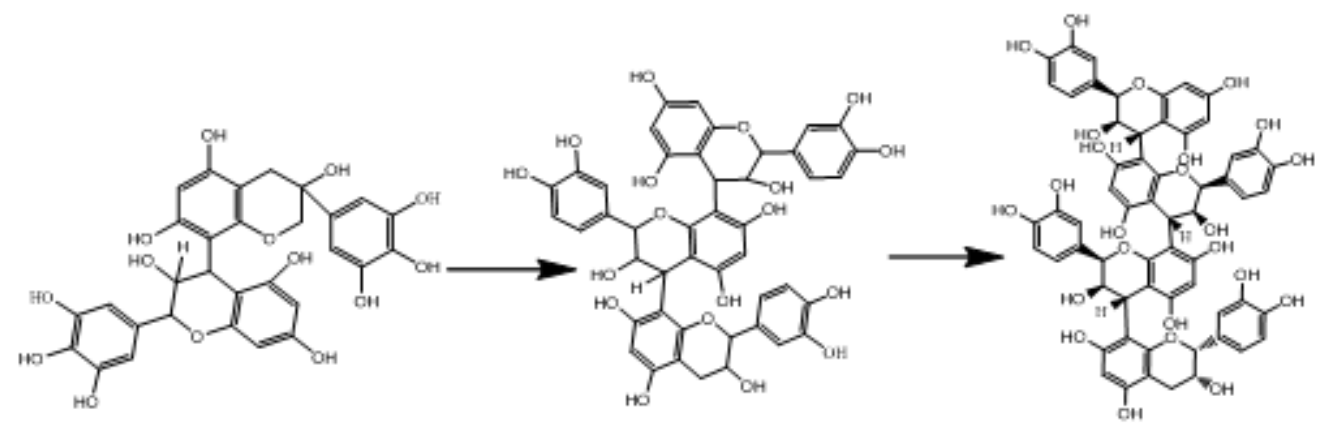

Dimer HOF $=-369.05 \mathrm{kcal} / \mathrm{mol}$ Trimer $\mathrm{HOF}=-414.35 \mathrm{kcal} / \mathrm{mol}$ Tetramer $\mathrm{HOF}=-490.18 \mathrm{kcal} / \mathrm{mol}$

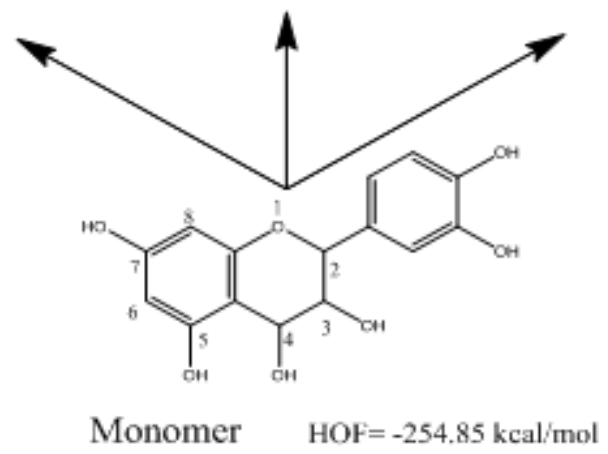

Figure 7. Structures of quercetin-metal complexes and their HOF values 
Black tea is the most popularlyconsumed tea in the world, and has excellent antioxidant activity (Tuet al., 2007). During the production of black tea, catechins are enzymatically oxidized to produce polymerized polyphenols including theaflavins and thearubigins. Theaflavins possess a benzotropolone skeleton that is formed from co-oxidation of appropriate pairs of catechins [27]. The major theaflavins in black tea are theaflavin (TF1), theaflavin-3-gallate (TF2A), theaflavin-3-gallate (TF2B) and theaflavin-3,3-digallate (TF3). On the other hand, thearubigins are largerand less well-characterized polymers. (Figure 8) [45]. Table 4 showed theHOF valueorderwas TF1 $>$ TF2 $>$ TF3. TF2A and TF2B had the similar HOF. The antioxidant dataof the four theaflavins determined by TEAC, HRSA and DPPH assays suggested that lower HOF value, higher antioxidant activity $[46,47,48]$. In addition, all the HOF values of theaflavinsis lower than that of EGCG $(-401.82 \mathrm{kcal} / \mathrm{mol})$, which is the strongest antioxidant among catechins. This result was in agreement with the previous report that theaflavins possess stronger antioxidant activity than catechins $[49,50]$. Theaflavins have two A-rings of flavanols linked by a fused seven-member ring. These structural features may provide more interaction sites with radicals. In addition, the benzotropolone moiety of theaflavins plays an important role in affording antioxidant protection for the preferred oxidation site, and might be responsible for electron donation because of the existence of resonanceforms [51].

Table 4. Antioxidant activities of various TFs in different assays and their HOF values

\begin{tabular}{ccccc}
\hline Theaflavins & ${\text { TEAC }(\mathrm{mM})^{\mathrm{a}}}^{\mathrm{a}}$ & $\mathrm{HRSA}(\mu \mathrm{M})^{\mathrm{b}}$ & $\mathrm{DPPH}(\mu \mathrm{M})^{\mathrm{b}}$ & $\mathrm{HOF}[\mathrm{kcal} / \mathrm{mol}]$ \\
\hline TF1 & $2.94 \pm 0.08$ & 3.9 & 33.2 & -415.70 \\
TF2A & $4.65 \pm 0.16$ & & & -547.83 \\
TF2B & $4.78 \pm 0.19$ & $3.1^{\mathrm{c}}$ & $10.9^{\mathrm{c}}$ & -549.64 \\
TF3 & $6.18 \pm 0.43$ & 1.6 & 7.7 & -680.27 \\
\hline
\end{tabular}

${ }^{\mathrm{a}}$ TEAC values (Miller et al., 1996); ${ }^{\mathrm{b}}$ Concentration of theaflavins for $25 \%$ inhibition of hydroxyl radical $\mathrm{OH}$. in non-enzymatic system $\left(\mathrm{IC}_{25}\right)$ and 50\% inhibition of DPPH radical ( $\mathrm{IC}_{50}$ ) (Yang et al., 2008); ${ }^{\mathrm{C}} \mathrm{TF} 2 \mathrm{~A}$ and TF2B mixture.<smiles>[R2]C1Cc2c(O)cc(O)cc2O[C@H]1c1cc(O)c(O)c2c(=O)c(O)cc([C@H]3Oc4cc(O)cc(O)c4C[C@H]3[R2])cc12</smiles>

Theaflavin

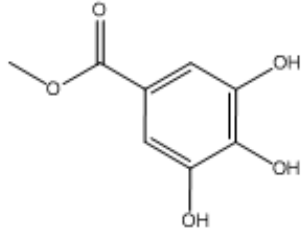

Galloyl group (GA)

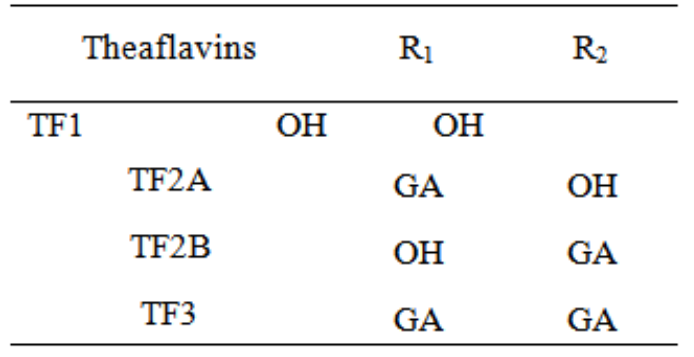

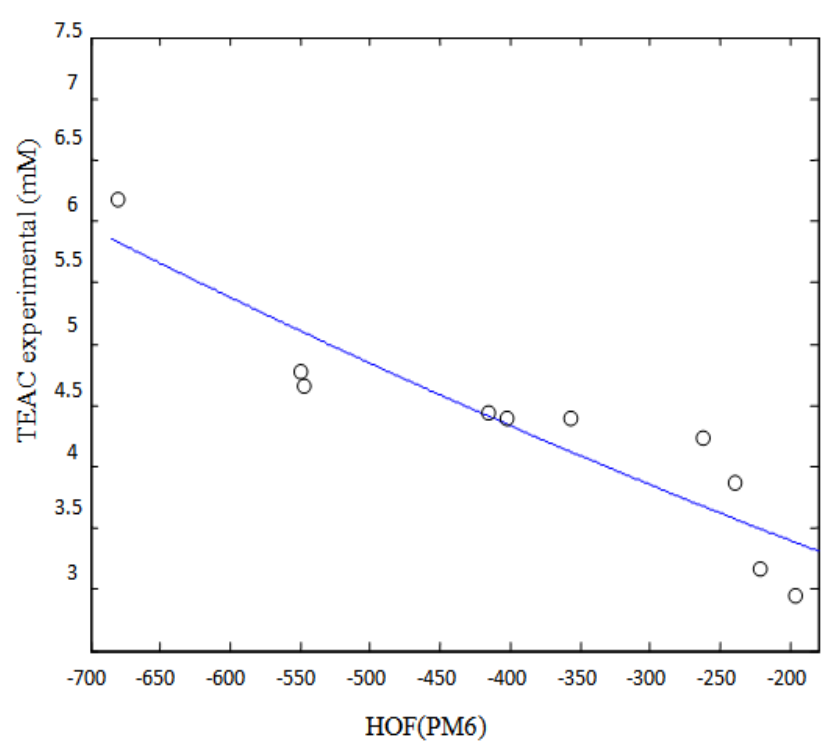

Figure 9. Scatter plot of calculated HOF value versus experimental TEAC values of 11 tea polyphenols (one outlier was omitted)

A scatter plot between the experimental TEAC values for ABTS radical and calculated HOF for a set of 11 polyphenols from Table 1, Table 2 and Table 4 is shown in Figure 9. From this set of TEAC data, the value of TF1 identified as outliers was omitted. A linear relationship indicated a general trend that higher TEAC is associated with lower HOF. A two-descriptor model is given by Eq. 2:

$$
\text { TEAC }=2.362-0.005 \mathrm{HOF} \quad \mathrm{N}=10
$$

The high correlation coefficient of fit $(r=-0.910)$ and the low RMS (0.12) confirmed the significance of this model, and indicated that HOF could be a viable tool for predicting the radical scavenging activity of tea polyphenols.

\section{Conclusions}

In this study, the relativity of the HOF value and the antioxidant activity of tea polyphenolsincluding flavanols, flavonols, flavanones, anthocyanins, proanthocyanidins and theaflavins, was analyzed by the PM6 method. It was found that the number and position of hydroxyl, and the structural groups influencing the conjugation between the B- and C-rings could affect the HOF value and radical scavenging activity of tea polyphenols. The HOF valuewas negatively correlated well with the antioxidant activity of tea polyphenols. These results suggested that the HOF value might be used to predict the antioxidant activity of polyphenols and other compounds.

\section{Acknowledgement}

This work was supported by Grant 2012T202-06 from Science Technology Department of Zhejiang Province, the National Science Foundation for Young Researchers of ZhejiangProvince (Grant No. LQ12C20004), and the Grant "Polyphenol enzymatic oxidation products" from Jianshixing Biotechnology R \& D co., LTD (Shanghai). 


\section{References}

[1] Cao, G. F., and Weng, X.C. , Study on oxidative stability of fish oil, China Oils and Fats, 20(4), 49-52, 1995.

[2] Costa, S., Utan, A., Cervellati, R., Speroni, E., and Guerra, M. C., Catechins: natural free-radical scavengers against ochratoxin Ainduced cell damage in a pig kidney cellline (LLC-PK1), Food and Chemical Toxicology, 45(10), 1910-1917, 2007.

[3] Speroni, E., and Scartezzini, P., Review on some plants of Indian traditional medicine with antioxidant activity, Journal of Ethnopharmacology, 71(1-2), 23-43. 2000.

[4] Phan, V., Nguyen, X. C., Nguyen, X. N., Vu, K. T., Ninh, K. B., Chau, V., Bui, H. T., Truong, N. H., Lee, S. H., Jang, H. D., and Kim, Y. H.,Antioxidant activity of a new C-glycosylflavone from the leaves of Ficus microcarpa, Bioorganic \& Medicinal Chemistry Letters, 21(2), 633-637, 2011.

[5] Chen, D. H., Chang, Q., Si, J. Y., and Shen, L. G., Research progress on natural polyphenol, Foreign medicine-medicinal plants fascicule, 12(1), 9-15, 1997.

[6] Chen, Z. Y., Su, Y. L., Xu, J. Z., Ng, C. H., Leung, L. K., and Huang, Y.,Antioxidant activity of tea theaflavins and methylated catechins in canola oil, Journal of the American Oil Chemists Society, 81(3), 269-274, 2004.

[7] Dobashi, Y., Hirano, T., Hirano, M., and Ohkatsu, Y.,Antioxidant and photo-antioxidant abilities of catechins, Journal of Photochemistry and Photobiology a-Chemistry, 197(2-3), 141-148, 2008.

[8] Haddad, E. H., Hudthagosol, C., McCarthy, K., Wang, P. W., Oda, K., and Sabate, J.,Pecans acutely increase plasma postprandial antioxidant capacity and catechins and decrease LDL oxidation in Humans. Journal of Nutrition, 141(1), 56-62, 2011.

[9] Olea-Azar, C., Folch-Cano, C., Jullian, C., and Speisky, H.,Antioxidant activity of inclusion complexes of tea catechins with beta-cyclodextrins by ORAC assays. Food Research International, 43(8), 2039-2044, 2010.

[10] Rivas-Gonzalo, J. C., Muselik, J., Garcia-Alonso, M., MartinLopez, M. P., and Zemlicka, M.,Measurement of antioxidant activity of wine catechins, procyanidins, anthocyanins and pyranoanthocyanins, International Journal of Molecular Sciences, 8(8), 797-809, 2007.

[11] Furuno, K., Akasako, T., \& Sugihara, N.,The contribution of the pyrogallol moiety to the superoxide radical scavenging activity of flavonoids. Biological \& Pharmaceutical Bulletin, 25(1), 19-23, 2002.

[12] Roginsky, V. A., Barsukova, T. K., Remorova, A. A., and Bors, W.,Moderate antioxidative efficiencies of flavonoids during peroxidation of methyl linoleate in homogeneous and micellar solutions, Journal of the American Oil Chemists Society, 73(6), 777-786, 1996.

[13] Kwak, S.Y., Yang, J.K., Choi, H.R., Park, K.C., Kim, Y.B., and Lee, Y. S., Synthesis and dual biological effects of hydroxycinnamoyl phenylalanyl/prolyl hydroxamic acid derivatives as tyrosinase inhibitor and antioxidant,Bioorganic \& Medicinal Chemistry Letters, 23(4), 1136-1142, 2013.

[14] Amic, D., Davidovic-Amic, D., Beslo, D., Rastija, V., Lucic, B., and Trinajstic, N., SAR and QSAR of the antioxidant activity of flavonoids, Current Medicinal Chemistry, 14(7), 827-845,2007.

[15] Maggiora, G. M.On outliers and activity cliffs - Why QSAR often disappoints, Journal of Chemical Information and Modeling, 46(4), 1535-1535, 2006.

[16] Dewar, M. J. S., Healy, E. F., Holder, A. J., and Yuan, Y. C.,Comments on a comparison of AM1 with the recently developed Pm3 Method, Journal of Computational Chemistry, 11(4), 541-542, 1990.

[17] Gundertofte, K., Palm, J., Pettersson, I., and Stamvik, A.,A Comparison of conformational energies calculated by molecular mechanics (MM2(85), Sybyl-5.1, Sybyl-5.21, and Chemx) and semiempirical (AM1 and PM3) methods, Journal of Computational Chemistry, 12(2), 200-208, 1991.

[18] Salahub, D., Rezac, J., Fanfrlik, J., and Hobza, P.,Semiempirical quantum chemical PM6 method augmented by dispersion and Hbonding correction terms reliably describes various types of noncovalent complexes, Journal of Chemical Theory and Computation, 5(7), 1749-1760, 2009.

[19] Amic, D., and Lucic, B., Reliability of bond dissociation enthalpy calculated by the PM6 method and experimental TEAC values in antiradical QSAR of flavonoids, Bioorganic \& Medicinal Chemistry, 18(1), 28-35, 2010.

[20] Zhang, H. Y.,AM1 Calculation of a parameter characterizing O-H bond dissociation energy, Chinese Journal of Organic Chemistry, 21(1), 66-70, 2001.

[21] Zhao, J. H., Liang, Y., and Yan, D. Y., Structure-antioxidation relationship of flavonoid antioxidants, Journal of North China University of Technique, 13(1), 36-44, 2001.

[22] He, B. H.,Some basic concepts of the quantum chemisitry. College Chemistry, 10(2), 50-54, 1995.

[23] Jiang, Y. S., Structural chemistry, Higher Education Press, Beijing, China,1997 47-49 (in Chinese).

[24] Zhang, H. Y., Structure-antioxidant activity relationships of flavonoids, Science in China (Series B), 29(1), 91-96. 1999.

[25] Nanjo, F., Goto, K., Seto, R., Suzuki, M., Sakai, M., and Hara, Y.,Scavenging effects of tea catechins and their derivatives on 1,1diphenyl-2-picrylhydrazyl radical, Free Radical Biology and Medicine, 21(6), 895-902, 1996.

[26] Cai, Y. Z., Sun, M., Xing, J., Luo, Q., and Corke, H.,Structureradical scavenging activity relationships of phenolic compounds from traditional Chinese medicinal plants, Life Sciences, 78(25), 2872-2888, 2006.

[27] Wan, X.C.,Tea Biochemistry, China Agriculture Press, Beijing, China, 2003, 18-32(in Chinese).

[28] Noda, Y., Kaneyuki, T., Mori, A., and Packer, L.,Antioxidant activities of pomegranate fruit extract and its anthocyanidins: Delphinidin, cyanidin, and pelargonidin,Journal of Agricultural and Food Chemistry, 50(1), 166-171,2002.

[29] RiceEvans, C. A., Miller, N. J., and Paganga, G., Structureantioxidant activity relationships of flavonoids and phenolic acids, Free Radical Biology and Medicine, 20(7), 933-956, 1996.

[30] Yang, X. Q., Tea polyphenol chemistry, Shanghai Science and Technology Press, Shanghai, China, 2003, 3-8,86-104(in Chinese)

[31] Husain, S. R., Cillard, J., and Cillard, P., Hydroxyl radical scavenging activity of flavonoids, Phytochemistry, 26(9), 24892491, 1987.

[32] Guo, Q. Q., Lin, S. Y., Li, D. G., and Huang, H. M. Research on antioxidation mechanism of dihydromyricetin, Food Science, 27(5), 121-123, 2006.

[33] Yang, Z. Y., Shang, Z. F., Wang, G. C., Yang, X., Pan, Y. M., and Zhao, X. Z. Theoretical study on the structure and strain of C-36, Acta Chimica Sinica, 59(3), 326-331, 2001.

[34] Yang, S. Z., Zhang, Y. S., and Peng, L. T., Antioxidative properties of dihydromyricetin, Journal of the Chinese Cereals and Oils Association, 19(2), 82-84, 2004.

[35] Bors, W., Heller, W., Michel, C., and Saran, M., Flavonoids as antioxidants - determination of radical-scavenging efficiencies. Methods in Enzymology, 186, 343-355, 1990.

[36] Jovanovic, S. V., Steenken, S., Hara, Y., and Simic, M. G. Reduction potentials of flavonoid and model phenoxyl radicals. Which ring in flavonoids is responsible for antioxidant activity? Journal of the Chemical Society-Perkin Transactions, 2(11), 24972504, 1996.

[37] Hayek, T., Fuhrman, B., Vaya, J., Rosenblat, M., Belinky, P., Coleman, R., Elis, A., and Aviram, M. Reduced progression of atherosclerosis in apolipoprotein E-deficient mice following consumption of red wine, or its polyphenols quercetin or catechin, is associated with reduced susceptibility of LDL to oxidation and aggregation, Arteriosclerosis Thrombosis and Vascular Biology, 17(11), 2744-2752, 1997.

[38] Jiang, L. Y., and Liu, Y. M.Study on antioxidative activities and quantum chemistry calculation of two quercetin-zinc complexes, Chinese Journal of Organic Chemistry, 25, 684-689, 2005.

[39] Zhou, J., Wang, J. Y., and Tang, N. Study on synthesis and antioxidant of quercetin-Cd and quercetin-Sc complex, Journal of Lanzhou University(Nature Science), 37, 123-125, 2001.

[40] Li, F., and Zheng, H. L., Study on fenton-methylene blue spectral analysis method for determining the inhibitory effect of quercetin complexes on hydroxyl free radical, Spectroscopy and Spectral Analysis, 26(12), 2294-2297, 2006.

[41] Bu, W. L., Research progress of the biological activity and mechanism of proanthocyanidins, Foreign medical science(section hygiene), 34(5), 311-315, 2007.

[42] Jordão, A.M., Correira, A.C., Relationship between antioxidant capacity proanthocyanidin and anthocyanin content during grape maturation of touriganacional and TintaRoriz grape varieties, South African Journal for Enology and Viticulture, 33(2), 214-224 2012. 
[43] Shi, B., Plant Polyphenol, Science Press, Beijing, China, 2000, 74-81(in Chinese).

[44] Tu, Y. Y., Yang, Z. Y., and Dong, F., Antioxidation mechanism of phenolic compounds in black tea and its structure-activity relatonship, Chinese Traditional and Herbal Drugs, 38(10), 15811585, 2007.

[45] Li, B., Vik, S.B., and Tu, Y.Y., Theaflavins inhibit the ATP synthase and the respiratory chain without increasing superoxide production, Journal of Nutritional Biochemistry, 23, 953-960, 2012.

[46] Miller, N. J., Castelluccio, C., Tijburg, L., and RiceEvans, C.,The antioxidant properties of theaflavins and their gallate esters Radical scavengers or metal chelators? Febs Letters, 392(1), 40-44, 1996.

[47] Yang, Z. Y., Jie, G. L., Dong, F., Xu, Y., Watanabe, N., and Tu, Y.Y., Radical-scavenging abilities and antioxidant properties of theaflavins and their gallate esters in $\mathrm{H}_{2} \mathrm{O}_{2}$-mediated oxidative damage system in the HPF-1 cells, Toxicology in Vitro, 22(5), 1250-1256, 2008.

[48] Yoshida, H., Ishikawa, T., Hosoai, H., Suzukawa, M., Ayaori, M., Hisada, T., Sawada, S., Yonemura, A., Higashi, K., Ito, T., Nakajima, K., Yamashita, T., Tomiyasu, K., Nishiwaki, M., Ohsuzu, F., and Nakamura, H.,Inhibitory effect of tea flavonoids on the ability of cells to oxidize low density lipoprotein, Biochemical Pharmacology, 58(11), 1695-1703, 1999.

[49] Tu, Y. Y., Tang, A. B., and Watanabe, N. The theaflavin monomers inhibit the cancer cells growth in vitro, Acta Biochimica Et Biophysica Sinica, 36(7), 508-512, 2004.

[50] Zhu, Y. X., Huang, H., Tu, Y. Y., A review of recent studies in China on the possible beneficial health effects of tea, International Journal of Food Science and Technology, 41(4), 333-340, 2006.

[51] Jin, D.Y., Xu, Y., Mei, X., Meng, Q., Gao, Y., Li, B., Tu, Y.Y.,Antiobesity and lipid lowering effects of theaflavinson highfat diet induced obese rats, Journal of functional foods 5, 11421150, 2013. 